\title{
¿IDENTIDAD POR VIAS DE DIVERSIDAD? \\ Actual perfil semiótico de identidad arquitectónica en Chile central.
}

Sofía Letelier P.

\begin{abstract}
Se aplica la realidad de la zona central del territorio chileno, apoyándose en el método semiótico para lenguajes no verbales, sobre un muestreo estadístico de campo, estableciendo indicadores de significación y comunicación entre legos y arquitectos. Detecta en la posible identidad arquitectónica de Chile Central una baja respuesta a los "principios de identidad", especialmente en lo concebido como "chileno" y en el grado de vocación a la producción de "objetos / edificios" con identidad. Demuestra que existe una distancia entre el campo noético del arquitecto y el campo lego lo cual lleva a reconocer un estado de incomunicación o que sufre fuertes interferencias.
\end{abstract}

A research was carried out in the Chilean central zone based on the semiotic method for non verbal languages using a field statistic sampling, thus stating meaning and communication indicators between laymen and architects. The research detects in the tentative Central Chilean Architectural Identity a low response to the "identity principles", especially as regards to those understood as "Chilean" and the degree of devotion to the production of "building-objetcs" with an identity. It shows that there is a big gap between the field of meaning used by an architect ("campo noético") and that of a layman which leads to a state of incommunication or at least shows strong interferences. Renouncing the pleasures of enjoying outstanding architectural works, it focuses on the identity phenomena from a global architectural viewpoint, as a globalising social expression where the architectural works represent the surrounding culture and it interprets deep feelings. This is an invitation to share a view of architecture from its class characteristics, even when the observer may have a different viewpoint.

\section{“PERFIL DE UN PERFIL DE IDENTIDAD"}

La Identidad -considerada como sello en lento hacer común; sujeta a recursos disponibles y validados en patrones colectivos- es un atributo natural y neutro que ha venido a cobrar signo de valor positivo para la arquitectura sólo en la segunda mitad de este siglo. Después de ingentes intentos en pos de innovación y redención que orientaron los diversos movimientos del siglo XX, emerge conscientemente en Chile como preocupación arquitectónica casi en los años '80, en medio de una permanente situación de apertura y valoración asignada en relación directa a la fidelidad del uso de modelos universales.

Para la Arquitectura había sido habitual asociar la Identidad con un cierto conservadurismo, en relación diacrónica de referencia al pasado, y con expectativas de cierta inercia de los rasgos que singularizan una determinada producción. Intentamos ahora, además, considerar la identidad como fenómeno sincrónico, donde se develarían procesos paralelos de significación y comunicación, que actualizan "de una determinada manera" códigos preexistentes, pero que también puede inducir a la generación de nuevos códigos: "particulares nuevos códigos» cuando existe identidad, como efecto tautológico de su propia presencia.

¿Qué factores concurren a modelar las singularidades que denominamos Identidad? Es posible que cualquier fuerza que deje huella en el proceso de significación de un colectivo: cualquiera que aporte contenido significativo; o cualquier hecho susceptible de ser "significado" para ser repetido. Pueden ser factor de identidad tanto los gestos arquitectónicos del pasado, como también una común respuesta al clima, a lo telúrico, a la economía o a la abundancia de un determinado recurso; alguna generalizada adopción de elementos prestigiosos -valorados en un momento y por algún motivo-; como asimismo elementos que llegan a ser emblemáticos o simbólicos por determinada coyuntura y que logran interpelar al común de una sociedad.

Hacer una descripción del perfil de la identidad de la arquitectura de Chile central (entre Ovalle y Concepción, con La Serena y Temuco como articuladores singulares), se dificulta si se abarcan en rigor los factores y condiciones a que el concepto mismo de identidad obliga. La amplitud del término puede ir desde un sentido de lógica objetual -«lo idéntico», «lo semejante" entre edificios, elementos o espacios-; hasta alcanzar diversos grados de actitudes empáticas de los individuos con los objetos, pudiendo llegar a vínculos de "apropiación» que llamamos «identificación». Ello invita a asumir una variedad de enfoques posibles para una descripción más certera. 
A partir de considerar la arquitectura como un lenguaje no verbal, el enfoque semiótico con que se aborda aquí la identidad arquitectónica 1 , es un punto de vista que por el hecho de manejar dos niveles de análisis vinculado de sus códigos —el «semático»sobre la forma y sustancia del signo; y el «noético», sobre sus contenidos significantes-, permite considerar tres situaciones correspondientes a sendos principios de identidad cultural, que dan cuenta de la extensión aludida:

a) Grado de identidad de los «objetos / edificios" que produce una sociedad (nivel semático):

- correspondencia de semejanza entre ellos,

particularidades reiteradas:

b) Grado de identificación de sociedad con "objetos/edificios" (nivel noético)en dos planos:

- "apropiación»o reconocimiento del objeto como propio

- "asociación» o vinculación (por otros) del objeto

con un determinado grupo:

c) Grado de vocación a la producción de «objetos / edificios" con identidad (conciencia de trascendencia), también en dos planos:

-demanda social de signos con identidad, hacia la identificación

- disposición de los autores para acoger significados colectivos antes que propios.

Por otra parte, si se atiende a la taxonomía analítica de los signos, - que primero distingue entre lo sustancial del signo y su código; para luego discernir niveles de independencia significativa desde el todo (sema), las partes que conservan significado (monemas), hasta los accidentes o características (llamados por nosotros "visemas" en lugar de los «fonemas" de la semiótica verbal)—, hemos distinguido dos planos de identidad: una *identidad estructurals (que apunta a la estabilidad de ciertas reglas globales) y una «identidad actitudinal» que responde a la operación más sutil sobre los accidentes.

La primera, dada por la convergencia objetiva en torno a signos y códigos a nivel sématico, más global y completo, que comprende la adscripción natural a determinados tipos, concepto de suyo estructural que no impide las variantes propias del acontecer y de la individualidad, pero que permite una interlocución fluida mediante estos lenguajes no verbales.

La segunda, "actitudinal», se expresa a nivel monémico -más elástico, propio del uso de elementos aislados-, los cuales, teniendo su propio significado. adecuan su sentido a su nueva situación de inserción. Y más propiamente a nivel visémico, que se expresa en compartir significados de los modos y accidentes, como pueden ser, proporciones, colores, materialidad, etc. Los principios y niveles de Identidad aplicados a una producción debieran formular un correlato semiótico, indicativo de su arraigo social.

\section{1. ¿IDENTIDAD POR "ANTI-IDENTIDAD"?}

Según este estudio, la producción arquitectónica de Chile central bajo el prisma descrito, se resiste a enmarcarse dentro de estos postulados de identidad. Antes bien, parece emerger cierta identidad chilena en lo que sería una "anti-identidad", pero que no adquiere aún el sentido contestatario y provocador que propuso la "anti-poesía", por ejemplo, chilena en origen. Si se acepta al menos que la identidad radica en algún grado de convergencia de rasgos (patrones) o sentido, la arquitectura chilena central tendería más bien por ahora a una dispersión divergente, sin clarificar aún su sentido.

\subsection{Estado General.}

Presencia de los Principios de Identidad.

Los principios deducidos como indicadores de identidad, parecen crecientemente vulnerarse en la producción arquitectónica de los últimos años en las principales ciudades de la zona

Respecto primero a la correspondencia por familiaridad, similitud, semejanzas, -en suma, patrones de unidad de los rasgos del lenguaje arquitectónico ambiente-, la coincidencia con este principio básico es escasa en la zona considerada y particularmente en cada ciudad. Del análisis cuantitativo no pudo deducirse la existencia de tipos claramente predominantes (semas); ni la reiteración de elementos hasta contribuir a una lectura unitaria (monemas); ni modos característicos que permitieran deducir un común sentido de valor en la caracterización de los accidentes (visemas). De allí que a nivel semático, podemos adelantar que tenderíamos a:

- identificarnos "por lo diverso"; no por "lo distinto" y singular -que sería "distintivo" y convergente-, sino por una gran gama de variaciones sin punto en común, a partir de escasos tipos a su vez divergentes en propósito $y$ origen.

Frente al segundo principio, el de la comunidad de significación e interpretación de los signos, -que implica un proceso socializado de asignación de sentido hasta llegar a que, tanto el grupo como sus miembros se sientan interpelados por los mismos estímulos, evocando coincidentes sentidos a los contenidos-, resultó que en la macrozona y en las localidades, no actúan filtros para definir un campo noético común. En un territorio que opuso su cardinalidad lineal a la comunicación e intercambio; con estratificación climática y pluviométrica perceptible, se aprecia adicionalmente cierto disvalor hacia la resultante de la masa arquitectónica heredada, que está marcada por la ausencia de preexistencias arquitectónicas indigenas y por condiciones desventajosas en cuanto a recursos y sismicidad. De modo tal que, por contraste, resulta explicable la adopción admirativa de expresiones importadas que compensan estos vacíos, hasta derivar en:

- identificación ( a nivel noético ) por valoración de lo diferente y nuevo, antes que de lo reconocible como propio.

El tercer principio, que condiciona la existencia de identidad a la vocación por su conservación y transferencia -en que resultaría comprometida inconscientemente el total de la sociedad (legos en general), y también quienes se abocan a la producción especializada ( los arquitectos) -resulta aún menos esclarecedor. Al ser contrastado el campo de repertorios significantes de los arquitectos - que está en la base de su orientación codificatoria-con el campo de repertorios significantes que dan sentido hoy a la decodificación lega, resultan campos dispares y con una muy reducida área de coincidencia. Ello indica que el proceso de significación /codificación del especialista y el proceso de significación /interpretación del lego, no se nutren de comunes contenidos ni existe una voluntad del especialista por develar el de aquel. La vocación de transferencia de una identidad - de generación en generación, o de propios a extraños-, se interfiere por otra personal que, por muy valiosa que se estime. subsume la identidad posible, dando como efecto: - vocación del especialista por expresar lo personal o una experiencia circunstancial en las obras, antes que contenidos con significado socializado.

Se concluye -en primera instancia-, que el proceso de identidad chileno estaría en un estado de dispersión, de apertura, de personalización, y distante aún de haber alcanzado 'lo distintivo','lo diferente', en un grado 'en que se difiere colectivamente' de otras expresiones arquitectónicas cuando hay Identidad.

\subsection{Potencialidad de un Estado}

\section{Actitudinal de Identidad.}

De acuerdo a nuestra distinción entre sidentidad estructural» $\mathrm{e}$ «identidad actitudinal - convergencia en la adopción de tipos y códigos solidarios, o en un nivel monémico/visémico, más sutil y que dice relación con lo cualitativo o modal-, se confirmaron señales de que la arquitectura de Chile central tendería a esta última.

Si bien la semática de la zona, no obstante adoptar una gama amplia de tipos, exhibe como se verá, cierta predominancia cuantitativa sólo de algunos (2) -lo que pudo llevar a inferir en una primera mirada la presencia de una identidad estructural-, el manejo de sus variaciones visémicas resultó tan libre y disperso que llevan al código de los tipos al límite de ser irreconocibles, desvaneciendo la lectura de identidad basada en el uso de tipos, especialmente hacia la 
actualidad. Por otra parte, no se advierte generación de tipos propios - ni a nivel de macrozona ni en localidades- que hubiesen aportado un indicador cierto de emergencia de identidad estructural.

Lo anterior no impide reconocer rasgos de «identidad actitudinal», la cual vendría dada por: a) Una cierta libel:ad semática y monémica (partes y elementos) desprejuiciada y evidenciada por el empleo de variados tipos en baja recurrencia, con o $\sin$ atinencia al código de inserción del modelo de origen, a referencias epocales $o$ al contexto asociable. Empleados más bien para nuevos usos o con propósitos alusivos a nuevos significados, invitan a un intencionado deslizamiento semántico en la lectura del edificio. b) Un manejo visémico (de cualidades) de amplia elasticidad, que pudiera deberse a la ausencia o debilidad de filtros culturales y físicos aludidos - del ámbito social, medioambiental, técnico, climático, geográfico, etc.-, de los cuales destacan: b. 1.) Inespecificidad y apertura tecnológica: la ausencia de preexistencias tecnológicas de saberes ancestrales; y la sismicidad propia de Chile que atentó contra la noción de perdurabilidad de las obras, llevan a una falta de filtro técnico cultural y a una actitud exploratoria. La no adscripción tecnológica específica $y$, por ende, la variada gama de proporciones dictadas por las diversas tecnologías, denota la ausencia de este factor como influencia posible en la identidad (Fig. 1) b.2.) Libertad frente al clima: el clima de la zona central, suficientemente templado y benigno en cuanto a temperaturas y humedad, ha devenido en extremo en un atenuante ( 0 no demandante) de mayores definiciones funcionales, formales, espaciales $y / 0$ tecnológicas. El clima no aparece en el nive consciente de los arquitectos y escasamente en el de los usuarios. Es muy significativo que la pluviometría que sí presenta amplias variaciones entre el norte y sur de la macrozona - no haya caracterizado tampoco a la arquitectura de ambos extremos, aunque en el 'imaginario' social - tanto del lego como del arquitecto, especialmente-, ésta impone una impronta para la zona centro-sur, que la arquitectura real no ha recogido.

b.3.) Proporciones ceñidas al rango de lo necesario: sea por sometimiento a la inmensidad de los "custodios" geográficos - mar y montaña-; sea por la experiencia de escasez dada por ser país remoto, o porque los recursos económicos no se prodigaron en la zona centro; o sea por la mencionada condición sísmica que conspira contra la «eternidad» de la arquitectura chilena, lo cierto es que las proporciones de los elementos, de los espacios y de las entidades como un todo, se atienen a lo necesario, cuando no «al mínimo necesarion. La ausencia de generosidad objetiva, hace por contraste aparecer entre las significaciones más preciadas del repertorio lego - no así en el de los arquitectos-, las de "cómodo" y "amplio", como altamente valoradas ( $\mathrm{o}$ añoradas).

c.) Una flexibilidad semántica: Además de lo señalado, los escasos patrones encontrados no develan tampoco una especial vocación por indicar los usos y funciones de los edificios y las tareas de sus partes. produciendo indefinición o flexibilidad semántica que aporta textos abiertos a diferentes lecturas. Se deja la esperada comunicación en una situación incierta, dada la baja especialización y especificación de los códigos propositivos que se manejan, como también subsecuentemente, de los que se requieren para traducir y decodificar.

Esta «ausencia de filtros heurísticos» -deducidos del modo divergente de abordar las situaciones descritas-, por haberlos evitado o no haberlos adoptado socialmente como tales, confirma de suyo una postura actitudinal social particular y potencialmente identificatoria de 'lo chileno', aunque no excenta de contradicciones:

Por un lado, apertura y desprejuicio - pero con empleo cauto- frente a la selección de tecnología (que en todas las culturas y épocas resulta central a la generación y resolución de un lenguaje); con una tendencia a la prescindencia o liberación, primero hacia los efectos del clima (factor frente al cual la arquitectura se ha propuesto desde antiguo ser límite, filtro o membrana metabólica como una de las razones de su existir) y luego a la connotación de función, son tendencias que augurarian expresiones de gran búsqueda y vuelo especulativo para la arquitectura chilena.

Por otro, asoma un ajuste y pragmatismo no ausente de inhibición dimensional - que al decir de Aguirre Silva (3), siguiendo a Spengler, sería un resultado cultural y contextual donde, en nuestro caso, el rol de la cordillera no puede ser desconocido-, que lleva a lo medido, a lo modesto, y a operar con lo justo como fuerte contrapeso a la grandiosidad que, al parecer, inhibe una posible audacia.

Esta dualidad se manifiesta también en otro campo de concretas actitudes dicotómicas. La rigurosa normativa sobre sismicidad y la frondosa reglamentación en pro de la privacidad; frente a la nula regulación sobre confort y calidad constructiva, por ejemplo, van dando un sello a la producción nacional. Lo anterior, sumado a condiciones de coyuntura, como son el mayor desarrollo actual, el crecimiento económico y el ilimitado dinamismo de la información - que aporta modelos lingüísticos en boga-. profundizan la dispersión e impiden resolver la contradicción advertida, perpetrando un estado de inequilibrio entre restricción y libertad, cuyo balance es necesario al desafío de la innovación con identidad.
La posibilidad de una poética de justo equilibrio entre la libertad y la cautela vislumbrada, implica un grado de dominio y maestría de quehacer en los modelos adoptados y en sus modos de uso, en fluida comunión semiótica entre arquitectos y sociedad, a partir del reducido imaginario y repertorio semiótico encontrado,

\section{REALIDAD / IMAGINARIO:}

\section{PATRONES DE IDENTIDAD}

Caracterizar la identidad de la arquitectura de Chile central como de una clase «actitudinal», sirvió para visualizar su sello general y no implica la inexistencia absoluta de códigos semáticos objetivos. El estudio, basado en la reacción de personas comunes y arquitectos en muestra aleatoria, arrojó algunos patrones generales o específicos locales -formados en la oferta de estímulos que lo construido propone como textos anónimos o intencionales de arquitectos pasados y vigentes-, y también un cierto perfil del sesgo semiótico de arquitectos y legos, no siempre coincidente.

Como indicadores se tomó las reacciones de apreciación hacia semas que, en anteriores estudios. resultaron ser altamente significativos en la decodificación interpretativa del texto arquitectónico: accesos, remates superiores y esquinas de edificios y. subsidiariamente, la envolvente, en cuanto a proporción relativa de llenos y vanos, materialidad y color. El perfil resultante se expone resumidamente.

\subsection{Patrón zonal de los Semas - Indicadores.}

Siendo la Identidad un aspecto cualitativo que adquiere sentido en su reiteración - dimensión cuantitativa-, es frente a la frecuencia y modo de esta reiteración que puede hablarse recién de identidad. Los indicadores mencionados no mostraron, en general, tendencias regionales o locales convergentes.

Coexisten numerosos tipos en uso -25 para accesos; 22 para remates superiores; y 20 para esquinas-, pero la frecuencia de uso de los tipos para cada rubro, por una parte, es tan baja, que no logran concitar unidad en el discurso global de cada ciudad o en la zona. La dispersión es más aguda en el caso de accesos y esquinas. Sólo en los remates se advierte alguna convergencia cuantitativa hacia un reducido número de tipos.

Por otra parte, el modo de empleo de los tipos es tan variado que su versión monémica o visémica hace a veces irreconocibles los que se reiteran. A diferencia de lo que sucede en la mirada cuantitativa, son ahora los «remates superiores" los que resultan más cualitativamente afectados por la versatilidad modal de los tipos, o al menos en ellos su mayor potencial 
comunicativo se advierte más resentido. Si bien puea ?n estar presente elementos asociables a determinados tipos, la aguda alteración de códigos llevaría - al deciı de L. Vaisman (4) - a sinsignificancias" (pérdida de significado); a sincomprensibilidades" (entorpecimiento de la lectura del texto para hacerlo comprensible); y a "perversiones significativas" ( ya sea que aporten una significación distante y contraria al sentido del texto, o bien que su inserción se haga ambigua y pierda significación al todo), etc. Ello desemboca en una comunicación ruidosa - o en incomunicación-y en un desperfilamiento de una posible identidad. Pocas coscis pueden afirmarse entonces como seguras, a nivel semático zonal:

a) Accesos: Los tipos más empleados se reiteran con una frecuencia poco incidente: $16 \%$ ( «perforación simple de la masa») y 7 \% («liberación de un módulo propuesto por la estructura»), ambos de baja intencionalidad y diseño, que en conjunto suman un 23 $\%$. Intermediando y también con baja incidencia. se encuentran dos tipos de ingresos protegidos ( «marquesina" o similar $15 \%$ y «hueco señalado por volumen saliente superiorm, en todas sus variantes $7,5 \%$ ), cuya suma, a pasar de representar también un $23 \%$ no logra concitar Identidad. El resto de los tipos se dispersa en cantidades inferiores al $5 \%$. Puede señalarse ademá que sólo un $40 \%$ de los accesos integra su sintaxis a la del resto del edificio o muestra intencionalidad en su relación con él.

b) Remates Superiores: Los tipos de remate cuya frecuencia individual pudiera considerarse de alguna incidencia por superar el $5 \%$, se polarizan en dos grandes macrotipos opuestos, como son: «techos de aguasw (31\%, donde un tercio está representado por «mansardas") y los remates "planos" $(38 \%$, con todas sus variantes, descle la «simple interrupción horizontal» hasta las cornisamentos y alerones). Por ser ambas tendencias contradictorias respecto al clima, contribuyen a una ambigüedad de la lectura del skyline. El resto de tipos si bien de mayor elaboración, articulación y adecuación a la arquitectura - tiene reducida presencia. Las tipologías predominantes mencionadas, presentan también escasa relación con la sintaxis general del edificio que culminan.

C) Esquinas: No aparecen como un motivo arquitectónico altamente diseñado. Cerca de un $42 \%$ se atiene al encuentro escueto de los planos de fachada concurrentes, ya sea por "arista simple" (26\%) o mediante "ochavo simple" (total o parcial, en un $16 \%$ ), sencillez que incide en el perfil perceptivo y, en sí mismo. pudiera sugerir una cierta lisura y austeridad. El resto, cercano al $60 \%$, presenta mayor elaboración pero conjuntamente a una fuerte dispersión en tipos, reforzándose esto por la importante presencia de lo que denominamos "esquina mixta" (13\%, que es no sólo «compuesta, sir o difícil de catalogar y describir). LO anterior hace de. dibujar nuevamente un posible perfil en esta vertiente : emática. Las esquinas más glaboradas, sin err bargo, son los únicos semas que integran de algún r lodo su texto al resto del edificio; jero su variedad, he ce difusa su incidencia en la Jinntidad del texto $u$ bano.

ذ) Envolvente, relo :ión relativa maza/vanos: Existiría ur.a tenciencia de las yersonas a identificarse levemente con envolve ntes en que predomina lo macizo (o bien lo cerrac 0 ), pero no se logra definir una determinada vocación visémica frente a los vanos o perforaciones (preterencia por determinados ritmos o proporciones. o por una mayor o menor contribución de los ritmos a la pregnancia del muro como tal). Más bien se advierte aceptación generalizada de una variedad aleatoria y de una baja integración de los vanos al discurso de! muro, cuando no una docilidad frente a los ritmos que modulo escuetamente la estructura soportante del edificio

En sintesis, la escasa codificación social constituiría nuestra sustancia plástica, base del posible Imaginario Zonal. La tencencia a preferir macicez de volumen, cuyo término o remate oscila en la dicotomía no resuelta se suma a indiferencia frente a otros semas y monemas que adquieren un discurso independiente en el texto.

\subsection{Patrones de Codificación del Uso} o Función del Edificio

En este país, escasos serían los códigos formulados para explicitar la «función/ destino» del edificio y, por ende, débil la habitualidad de las personas a la interpretación de claves que interpelen hacia una decodificación compartida de lo que es cada edificio A pesar de ello, pueden mencionarse rudimentos que orientan la lectura urbana, (lectura que no siempre resultó certera, atribuyéndole destinos erróneos) a) Lo habitacional: Discriminarlo de lo que no es, parece ser lo único efectivo —de tipo categorial-, y se basa en rasgos gruesos que se le atribuyen socialmente: - Remate por "aguas" o "mansarda";

- Cierta macicez (relativa) o hermeticidad del muro de fachada.

b) Lo institucional: en cambio, genéricamente considerado ( «Servicio», «Poder/Gobierno»), se decodifica globalmente por un rasgo único, que cuantitativamente no corresponde con la realidad:

- Remate superior horizontal, simple o con cornisamento.

En menor medida -y por claves de tipo monémico o visémico, ya que no pudo establecerse para ello tipologías semáticas-, se esbozan patrones frente a destinos como "oficinas" (hermetismo, regularidad de tamaños); "comercio» (regularidad,
Tecnologia común que da unidad a cerros de

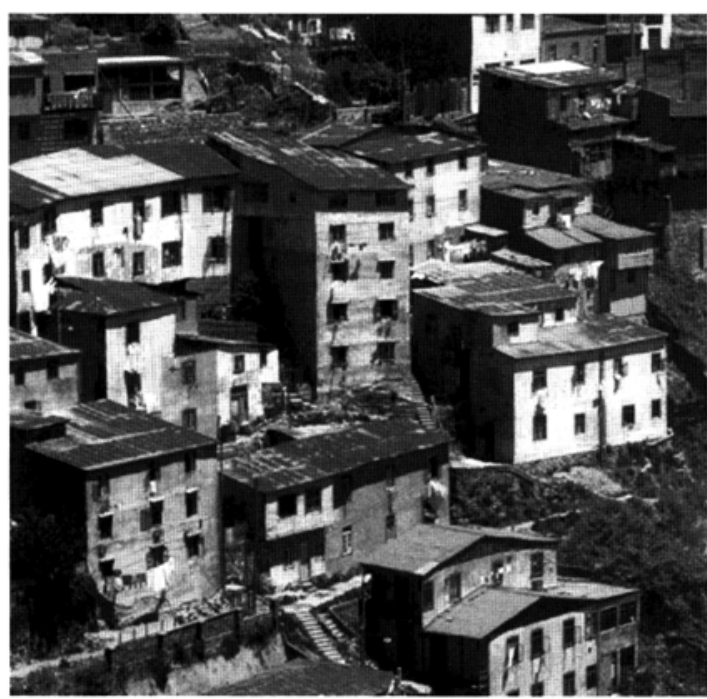




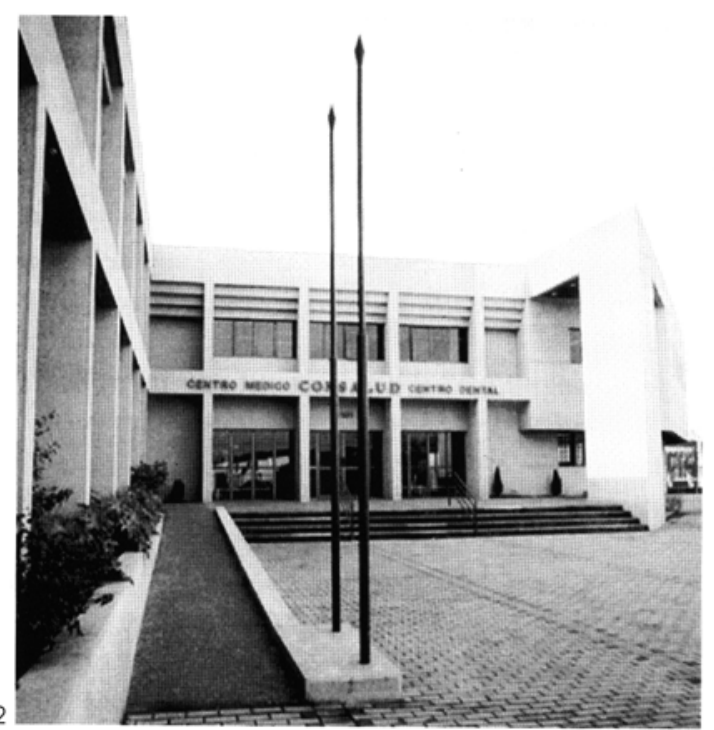

apertura); salud ( blanco, de estructura evidente y repetitiva). Otros destinos no han logrado conformar imaginario (Fig 2.)

Vista la Comunicación del Uso (o destino) del edificio como «sistema de significación/comunicación entre legos y arquitectos, puede resumirse diciendo que: i. No se cierra el circuito significacióncomunicación respecto al destino del edificio, aunque hay un grado de comunicación que es débil.

ii. Existiría un «subsistema de significación» en cuanto a lo habitacional -con reconocimiento compartido (en la escala de media y baja altura), y que es pertinente en gran medida-y uno, más limitado, en cuanto a lo institucional (encontrándose incluso "códigos anquilosados», como es el caso de edificios de salud). iii. Ambos grupos manejan mejor códigos unifuncionales o específicos; tienen dificultad de lectura de códigos mixtos.

iv. Se debilita el «sistema de comunicación», dado que existiria:

- hipercodificación de modelos externos por parte de arquitectos, no adaptados a las particularidades y finuras que el lego percibe:

- hipocodificación de lo universal por parte de lego, a quien la variedad hace laxa la percepción y hace aceptar lo inespecífico existente como "adecuado", dejando a la arquitectura percibida más flexible y permeable a sus propias vivencias.

\subsection{Patrones Estético-Formales.}

Orientación afectiva.

Por alguna razón, los patrones estéticos que se manifiestan en orientaciones afectivas positivas de valoración en el Imaginario Lego, no coinciden con la arquitectura que se produce, en general. Pudo aislarse sólo algunos patrones con cierta certeza:

a) Accesos: se valora su «espacialidad” (definición. cobertura, generosidad) (Fig. 4)

b) Remates Superiores: se valora la «reiteración de lo habitual o contextualy.

c) Esquinas: «macizas" para vivienda y; «articuladas o aéreas» para otros usos.

d) Envolvente: se valora, en general, los «énfasis explícitos $n$ :

- sintaxis clara y adaptada entre los diversos semas:

- «macicez o cerramiento» del muro:

- "pregnancia" global o parcial (con excepción de cilindros):

- "colorido neutro o conservador", en lo institucional;

- "colorido cálido» (y poco saturado) en lo

habitacional:

- "madera” en detalles, sólo a nivel monémico.

Revisadas las verbalizaciones que acompañan las preferencias, dando origen a la deducción de estos patrones estéticos; y puestos en una visión sistémica - como «sistema de significación/comunicación» valorativo afectivo- se pudo establecer:

. Hay un único «subsistema de significación” estético positivo que llega a "comunicación», dado que lo comparten arquitectos y legos. Gira en torno al concepto "clásico" explicitado en el término «elegante" (uno de los escasos adjetivos positivos que resultaron verbalizados por los arquitectos) (Fig. 3).

ii. Un "subsistema de significación" que es coincidentemente negativo para ambos - por lo que alcanza "comunicación" - en torno a signos que evocan "frí/feo/ encerrado", (nótese el importante campo de coincidencia).

iii. Existe un claro «subsistema de significación» entre legos, en torno a los conceptos «moderno/austero/ acogedor", que se superpone al significado "cómodo" / funcional, amplio (los arquitectos encuestados no verbalizan este tipo de adjetivos)

iv. Hay un definido «subsistema de significación tácito entre arquitectos, que no alcanza "Comunicación»: los contenidos estéticos positivos, no se explicitan fácil y verbalmente por ellos. Cuando ocurre, no se refieren habitualmente a una condición espacial, sino formal; más específicamente, a sinadecuación» formal. (Por ser «anti», no pudo establecerse los componentes de este «subsistema significativo del especialista»). Se constituye entonces un «subsistema» en lo netamente visual entre arquitectos, deducido a partir de sus referencias a la forma, y a que evita minimiza - o incluso peyora- juicios que involucren sensaciones.

v. El «subsistema significativo» del lego es más pluridimensional y contiene conceptos vivenciales especialmente-, tanto como espaciales, formales y funcionales. Su significación incluye la «sensación» como vinculante de sus juicios.

vi. Existiría un «subsistema significativo" del ornamento, que no alcanza comunicación: mientras para el arquitecto es «significativo» -en el sentido estricto de que "significa determinado algo»-, y busca su pertinencia, para el lego se acepta abiertamente sin significarlo (resultando accesorio).

Podemos agregar otras dos verificaciones globales respecto a lo estético:

- El "sistema significativo estético» de arquitectos, en general "adapta» modelos y tipos, inducido por la situación de las obras y, para verbalizar sus impresiones, asocia "casos" o ejemplos (en alusiones), antes que «reglas"

- El «sistema significativo estético" del lego, como conjunto, tiende a una aceptación de los estímulos y "adopta” las incoherencias sintácticas, con un umbral muy amplio, lo que indica un estado de baja expectativa respecto a la arquitectura.

La coincidencia en los dos primeros subsistemas 
enumerados que llegan hasta la "comunicación» - - o "elegante" y un amplio "feo"-, permiten vislumbrar un camino

\subsection{Patrones perceptuales de "lo Chileno".}

Los rasgos que colectivamente se "significan" y reconocen como "chilenos», no son estructurales. Son visémicos y actúan en conjunto: material, color, escala, tamaño y relación proporcional lleno/vacio.

El «proceso de significación» que concluye en esta asignación semántica fue difícil de establecer, y más aún el por qué este reducido espectro de patrones apela a lo nacional conformando un Imaginario de "Chilenidad" que cuantitativamente dista de coincidir con la realidad. Los patrones resultaron ser:

a) Accesos: con "protecciones" y "portadas" especialmente del tipo «neocolonial

b) Remates Superiores:

- "techos de aguas" (asignados desde Santiağo a sur):

- "terraza/ pérgola” ( iquiqueña, se asume desde santiago al norte):

- "mansardas" (con gran elasticidad de código y ubicuidad):

c) Esquinas: ninguna en particular, sólo efectos de macicez;

d) Envolvente: masividad, con variedad e integración aleatoria de vanos.

Estos "patrones de chilenidad" no llegan a defini con claridad "Subsistemas de significación», aunque diremos en general que:

i. Para ambos - legos y arquitectos - estos patrones no singularizan, antes bien, «acogen» una mezcla de "claves climáticas" ( código por ausencia).

i. Para ambos también, «las no estridencias» ni acentuaciones exageradas en cada sema o en la interrelación de material, color, escala y tamaño, señalan «lo chileno".

\subsection{Patrones de respuesta al Climo.}

La habitualidad opera para hacer que no se reconozcan ciertas respuestas al clima como claves de identificación de lo propio. Sólo algunas significancias a nivel monémico o visémico, pueden encontrarse. Los semas por separado - accesos-, remates y esquinasno fueron inductores por sí solos de asignación a pertenencia climática, pero de la preferencia por ellos pueden deducirse algunos efectos inductores:

a) Accesos: Las "protecciones a la lluvia" son un primer inductor de significado, antes que los indicios de "seco". Es el caso de los "pórticos", "techos de aguas", etc., que sobreponen su significado a cualquier otro presente que aluda a situaciones más secas y benignas. (Como en las combinaciones "edificio techo planow + "pórtico de acceso"= se asume «lluvioso"). b) Envolvente: El tamaño de las ventanas es «ubicuon: no se le asigna "pertenencia" en relación al control de temperatura, por ejemplo, o por mayor o menor necesidad de luz. Sólo el color es relacionado a "clima» (excepto en Temuco, que difiere):

- Colores claros $=$ «norte

- Colores obscuros/ ladrillo $=$ «sur».

De lo anterior puede deducirse la existencia, aunque difusa y con escaso grado de comunicación entre legos y arquitectos - de un «sistema de significación» global, que responde sólo a una muy gruesa "cardinalidad" ( sur= lluvioso, oscuro; norte= seco,luminoso), que la cuantificación de la realidad contradijo:

- el norte: refleja más al Imaginario descrito,

- el centro: no ha formado un Imaginario climático, - el sur: responde en escasa medida al Imaginario atribuído.

2.6. Patrones aportados por la Geografía

Los patrones dictados por lo geográfico - tal como la geografía misma-, son definidos y polares, pero sin asiento tampoco en la realidad arquitectónica

Se dio una distinción entre «arquitectura de costa (con una significación clara), respecto de la «de valle» (sin significación), y «de precordillera" ( con significación difusa). Semas específicos no se detectaron al respecto, pero sí integraciones de éstos con monemas o visemas propios para estas situaciones:

Un subsistema de significación "costa", resultó comunicativo ya que es compartido por legos y arquitectos, y está constituido por «liviandad/ movimiento/ colores claros y blancon, (para los legos se integra, además, por «neocolonial»).

ii. Un subsistema de significación para «precordillera». que alcanza comunicación entre legos y arquitectos aún en su esterotipo-y comprende: «techos de aguas»/ color oscuro/ ventanas pequeñas / madera.

Tampoco los ríos urbanos - como los de Santiago o Concepción-, u otros accidentes logran significar a la arquitectura de los lugares en que se presentan.

2.7. Patrones de valoración e identificación epocal. Las claves de orientación epocal que emplea el chileno de hoy, resultan altamente decidoras del efecto que la arquitectura proporcionada ha ejercido. Ellas son:

a) Accesos: articulación; sobreposición de lenguajes: estrechez; mezcla de elementos; «agregación» (antes que adecuación):

be Remates Superiores: cualquiera, si posee proporciones económicas; el tipo "placa suspendida" ( en cualquier caso) y la "mansarda" (en cualquier caso, incluso "alusión mansarda", muy lejana al modelo). ( Figs. 5,6,7);
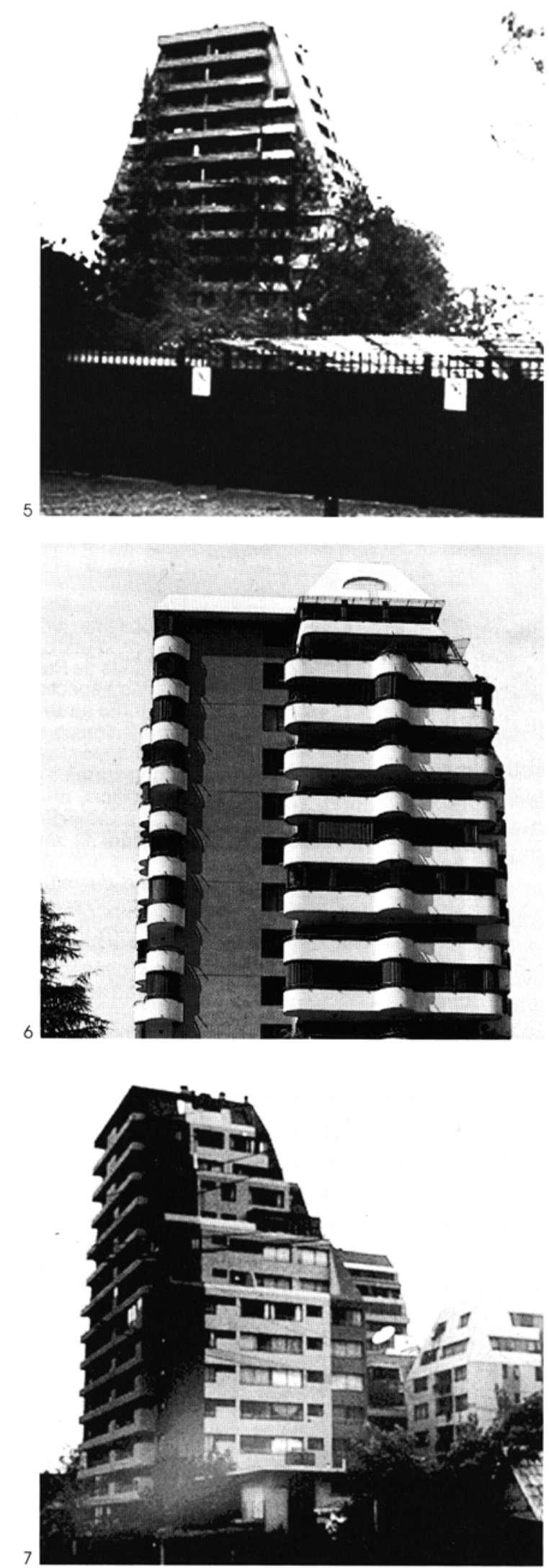

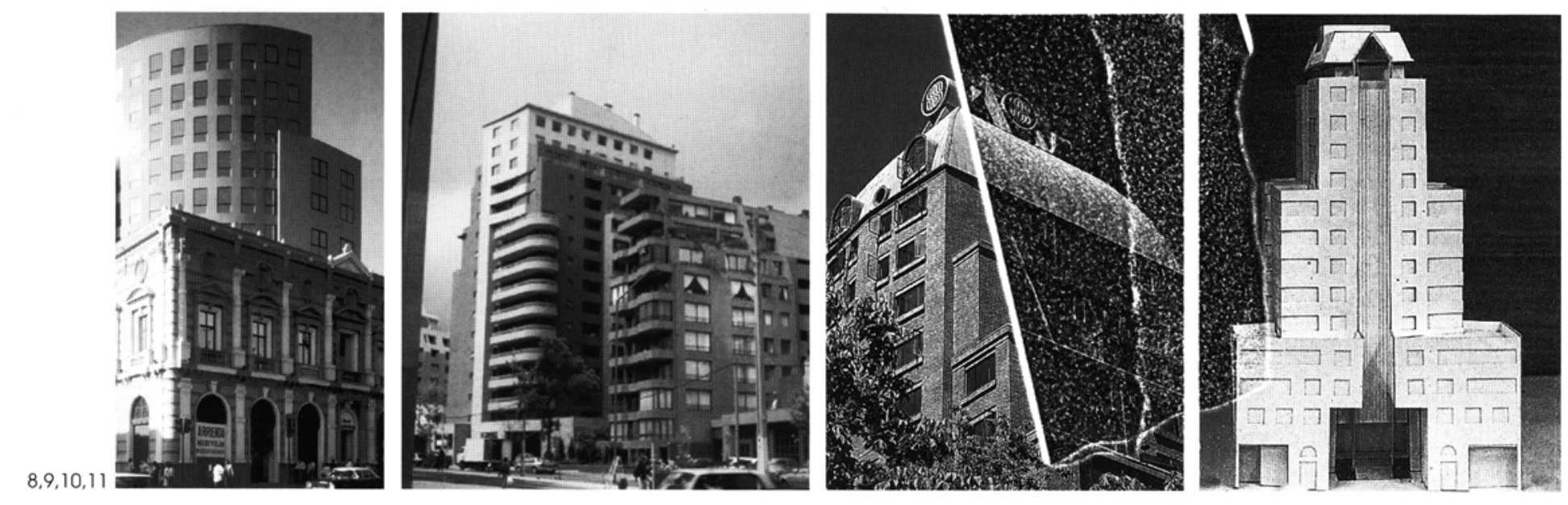

c) Esquinas: desagregación (excepto para personas de La Serena y Temuco ):

d) Envolvente: xladrillo aparenten; ventanas variadas; intervalos no definidos; proporciones poco generosas; agregación de monemas descontextualizados. Colores blanco o cálidos apastelados.

Lo señalado -extraído de la atribución de "reciente" $y$ "chileno», sobre una muestra de 52 imágenes- constituye, por sí mismo, un sistema completo de significación/comunicación entre legos y arquitectos, por ser éstos quienes originan las reiteraciones desde donde se extraen los códigos del Imaginario Lego. (Si bien el patrón de «lo reciente” coincide en toda la zona, es menor en ciudades grandes, donde se individualiza lo nuevo con dificultad o no se reconoce. Esta relativización puede deberse a que, siendo estas ciudades de mayor tamaño, la variedad de oferta lingüistica - con «revivals» que confunden incluso a arquitectos-, aumenta la desorientación decodificadora).

La arquitectura más antigua se valora incondicionalmente si posee riqueza ornamental y coherencia interna, aun en estado de máximo detrioro. Ello confirma una valoración antitética y una incomunicación de este lenguaje no verbal en la oferta de textos de hoy

\subsection{Patrones locales de algunas}

\section{ciudades centrales.}

Fue posible recoger ciertos rasgos que esbozan alguna «identificación local», en la dimensión de autoimagen y en la dimensión de reconocimiento o asignación de imagen por ajenos, aun cuando estos imaginarios no correspondan siempre con la realidad cuantificada. La tabla adjunta (ver T. 1) incluye. resumidamente, los únicos patrones de imagen local que pudo verificarse: el «imaginario» se conforma a partir de la realidad, y se puebla de patrones aprendidos de un creer colectivo que atribuye al clima mayor importancia que la que la práctica concede.

\section{ARQUITECTOS: ACTITUDES Y} "PATRONES DE IDENTIDAD" HOY.

Sabemos que los arquitectos chilenos ensayan con sostenido ahinco una expresión propia, en una incesante búsqueda de lenguajes para establecer la necesaria y eficaz comunicación con el usuario previsto o accidental, y para expresar lo que a su juicio es la «identidad sentida" de un determinado lugar.

A juzgar por los resultados expuestos, la Identidad de lo chileno se muestra esquiva para develarse a los esfuerzos individuales que se hacen para asirla, a pesar de lo cual, la suma de acciones ha producido efectos $y$ conformado patrones involuntarios. Este repertorio que desplegamos aquí, representa al menos parte de Nuestra Identidad de Chile Central —a partir de una investigación inductiva y abierta-, tiene al menos el mérito de proponer bases para una discusión.

Desconocemos cuál puede ser la reacción de la generalidad de los arquitectos al momento de acceder al hecho de que existen patrones comprobados de comportamiento decodificatorio por parte de los legos frente a la arquitectura. No sabemos tampoco si la existencia de estos patrones demandaría una postura ética consecuente - constituyendo un desafío- 0 , por el contrario, ellos deben permanecer como una información más, dado que vulneraría, de algún modo, la libertad creativa que todo arte con celo reclama. Sin embargo, comprobaciones hechas por el equipo induciendo situaciones de proyecto - de reciclaje con resemantización, para arquitectos jóvenes; con y sin antecedentes del repertorio del Imaginario Lego

\begin{tabular}{|c|c|c|c|}
\hline CIUDAD & AUTOIMAGEN LOCAL & 'IMAGINARIO' EN LA ZONA & PERFIL REAL ESTADÍSTICO \\
\hline LA SERENA & $\begin{array}{l}\text { consistente y coincidente con lo } \\
\text { real }\end{array}$ & $\begin{array}{l}\text { coincidente, con lo real, es } \\
\text { reconocido por ellos }\end{array}$ & $\begin{array}{l}\text { versión local de 'colonial'; remates } \\
\text { de aguas }\end{array}$ \\
\hline VALPARAÍSO & $\begin{array}{l}\text { identifican su ciudad } \\
\text { consistentemente con la realidad }\end{array}$ & $\begin{array}{l}\text { idealizado, en 'antiguo' } \\
\text { 'fragmentación' y 'remates de } \\
\text { aguas' }\end{array}$ & $\begin{array}{l}\text { predominio de 'remates planos' } \\
\text { con cornisamento }\end{array}$ \\
\hline TALCA & no confirmada & $\begin{array}{l}\text { no se reconoce: sólo se recuerda } \\
\text { su escala media }\end{array}$ & $\begin{array}{l}\text { remates planos; colores claros, } \\
\text { calidad hermeticidad. }\end{array}$ \\
\hline CHILLÁN & $\begin{array}{l}\text { se atribulle rasgos propios que no } \\
\text { identifican excepto los 'accesos } \\
\text { protegidos' }\end{array}$ & $\begin{array}{l}\text { no se reconoce; sólo se recuerda } \\
\text { su escala media }\end{array}$ & $\begin{array}{l}\text { remates planos: colores claros; } \\
\text { expresión del Mov. Moderno; }\end{array}$ \\
\hline CONCEPCIÓN & $\begin{array}{l}\text { autoimagen contradictoria o } \\
\text { ausencia de autoimagen }\end{array}$ & $\begin{array}{l}\text { no se reconoce; se le atribuye } \\
\text { colorido oscuro, compacidad, } \\
\text { estilo 'tardomoderno' }\end{array}$ & $\begin{array}{l}\text { remates planos; colores claros, } \\
\text { accesos protegidos. }\end{array}$ \\
\hline TEMUCO & & $\begin{array}{l}\text { idealizado: -'madera'; } \\
\text { - 'color oscuro' } \\
\text { - 'techo aguas'/mansardas; } \\
\text { aleros y protección a lluvias }\end{array}$ & $\begin{array}{l}\text { - remates planos, sin mansardas } \\
\text { (hasta '93) } \\
\text { - colores claros: } \\
\text { - escasa protección en accesos. }\end{array}$ \\
\hline
\end{tabular}

descrito-, indican que entre los jóvenes hay potencial de apertura a 'códigos no propios': no resultaron indiferentes ni voluntaristas, especialmente frente al posible «transeúnte» (externo a la obra),más que ante un posible 'habitante'

Esto hace promisoria la existencia de una Identidad emergente, aunque la última constatación el privilegio hacia el transeúnte- nos suma nuevamente en perplejidad: una arquitectura que se empeñaría en lo externo.

\subsection{Persistente Incomunicación en la Identidad Emergente.}

Entre los rasgos emergentes de Identidad Arquitectónica Chilena, hemos señalado como constituyentes de comunicación a aquellos coincidentes entre legos y arquitectos. Otros son propios de cada estamento y alcanzan sólo a conformar «subsistemas de significación»; y, por último, ciertos rasgos son francamente opuestos, que se interpretan como incomunicación de los interlocutores o efecto interfiriente del mensaje.

Más allá de los significados objetivos detectados, la «actitud apreciativa" que el instrumento permitió detectar, indica que es más honda la incomunicación cuantificada en primera instancia, pues se vio que en varias de las coincidencias entre legos y arquitectos, las "motivaciones» que las originan difieren fuertemente. Es decir, no obstante otorgarse igual valor apreciativo al signo, ello respondía a un diferente significado. Los principales desencuentros advertidos serian:

i. Mientras los legos explicitan su sentir, los arquitectos adoptan tres actitudes:

- eluden el juicio crítico con escamoteos:

- hacen juicios neutros, escudándose en la objetividad del especialista:

- emplean expresiones lúdricas (en tanto tal inobjetables)

ii. Cuando hay valoración positiva común, difieren las motivaciones (con excepción de los enunciados de tipo "clásico», que ambos positivizan por igual, en razón de «elegancia», "Claridad», etc. (Figs. 3 y 11 ):

- el arquitecto da valor por visualidad o estatus del contexto;

- el lego da valor a lo vivencial o funcional.

iii. Cuando hay valoración de lo pregnante, si alude a un objeto:

- mientras el lego hipo o hipercodifica hacia el referente sugerido;

- el arquitecto inhibe la asociación al referente o la "negátiviza"

iv. Cuando hay valoración negativa común frente a una hibridación:

- el lego la rechaza por sí misma

- mientras el arquitecto condiciona el rechazo a la situación. 

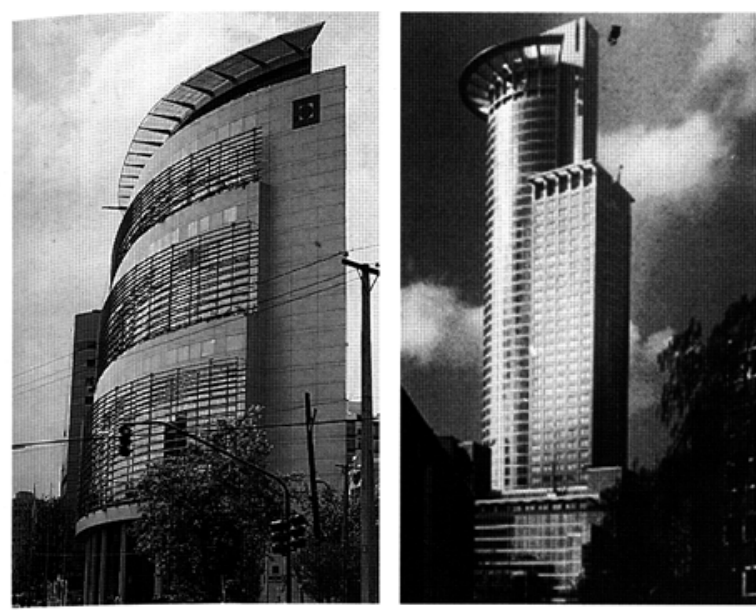

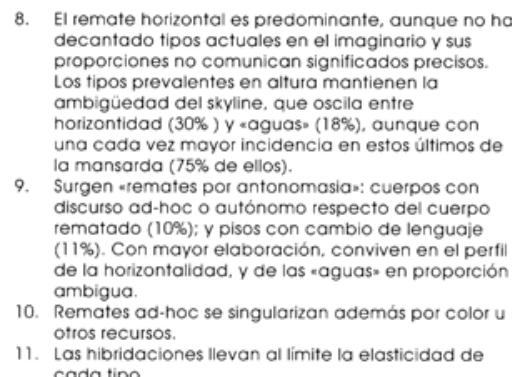

cada tipo. v. Cuando hay común visión negativa de lo formal:

- el lego la rechaza si atenta a lo vivencial io

funcional;

- mientras el arquitecto lo hace por códigos

formales visémicos.

vi. Cuando ambos emplean hipo o

hipercodificaciones:

- el lego las usa como recurso positivo (lo que no puede explicar):

- el arquitecto expresa así lo negativo ( se niega a explicitar, ridiculiza):

Que las coincidencias se refieran a aspectos tan puntuales y, aparentemente no conectados, no obedece a un devaneo del estudio, sino al hecho de que, por ser una investigación abierta, se descubrieron múltiples facetas aun cuando sólo se consignan aquí aquellas cuyo resultado resultó «significativo» en sentido estadístico. Su conjunto, sin embargo, creemos que esboza bien un perfil bastante nítido.

\subsection{Ilustración: Perfil de los Remates}

\section{en edificios recientes de altura.}

La muestra del perfil que han adquirido los remates de edificios en altura de los cinco últimos años, hizo posible confirmar lo antes dicho. Se eligió este nivel semático, por ser 'inescapable': presencia y referencia inevitable para el transeúnte e instancia obligada de definición y término de texto para el discurso arquitectónico

La tendencia que se observa, visto cuantitativamente, indica que la dispersión tipológica va en aumento, no por el número de tipos - que disminuye de 22 a 9 - sino por la cantidad de versiones, la cual aumenta hasta 7 por tipo.

Vista cualitativamente (Fig. 8 en adelante), se mantienen la ambigüedad del skyline entre "horizontalidad" $y$ «aguas", de las cuales un $75 \%$ son mansardas; surgen "remates por antonomasia" o cuerpos con discurso ad-hoc, cúpulas, bóvedas y otros cuerpos que aportan su propia geometría autorreferente; cuando se adaptan a la altura tipologías preexistentes, las proporciones de los tipos usados sobre catorce pisos, no dan cuenta del alargamiento del cuerpo o fuste que rematan. Lo anterior es especialmente evidente en el caso de antetechos y mansardas, empleadas con gran elasticidad de proporciones. Adicionalmente al uso elástico de la mansarda, la restricción normativa por «rasantes»dictadas por el derecho al sol-, ha propiciado diversos tipos de «escalonamientos» o cortes «alusivos a mansarda en la decodificación visual — «telescópicos»; "pirámides irregulares»-, y diversos modos de truncamiento de prismas, todo lo cual contribuye a hacer más heterogéneo el perfil.

Desde el punto de vista de la Teoría de la
Creatividad, si bien el tratamiento en altura de los remates horizotales $\mathrm{y}$ "de aguas" -incluidas las mansardas cuando se atienen al tipo- constituirían sólo evoluciones o transformaciones en un nivel primario de creatividad, el tratamiento de muchos de los «remates ad-hoc» y de los truncamientos («lustrines") serían una forma de innovación -o emergencia de invención-. por ser soluciones únicas, sin antecedentes en otros paises. Ello, que es propio y que pudiera contribuir a una identificación $-y$, por tanto $a$ *identidad - , más bien la dificultan dado que por ahora se multiplican con tal variedad, que producen un efecto entrópico. Es posible que estos recursos puedan llegar a depurarse y perfeccionarse como retórica local.

Pero, para la edificación en altura, la incidencia en la Identidad de un determinado rasgo en la comunicación que oferta el paisaje, no está sólo relacionada a la recurrencia. La incidencia cualitativa en la identidad depende también de la pregnancia y elocuencia del signo; de su estructuración (inducción de ejes, centros o pautas de lectura), en fin, de su capacidad de concitar atención urbana e interés suficientes por el tiempo necesario para captar su código y decodificarlo. En nuestro paisaje, si bien están adquiriendo gran incidencia las denominadas «remates ad-hocm, las cuales, como cuerpos de geometría propia, articulan y atraen hacia su propio texto, interfiriendo la lectura de otras tipologías de menor fuerza que condicionan su comunicación urbana a la existencia o no de 'ruidos visuales'. Así, el «remate horizontal» $-\mathrm{o}$ el tendiente a él一, aún redundante, es de poca incidencia en la Identidad de altura por cuanto su manejo local carece de tensión suficiente para imponerse, al minimizarse en general las proporciones del elemento borde (dejándose justo en «lo necesario»), o al seccionarse su lectura con múltiples recursos "amortiguadores».

Sin aplicar juicios de valor, si bien en la edificación en altura se detectan rasgos que pueden derivar en identidad, su análisis no permite afirmarla en definitiva.

\section{REFLEXION}

La dispersión aparente de lo expuesto, que se debe a la naturaleza multifactorial y compleja de la identidad, invita a reducirlo a la sensación que nos queda, a partir de algo dicho en la segunda parte: a) que no hay convergencia de los propios arquitectos en lo que les gusta:

b) que los legos sí coinciden en aquello que les gusta y

c) que la escasa convergencia de ambos se encuerntra en lo que les disgusta, ...estaríamos en presencia de un «ideolecto», algo hermético por parte de los arquitectos, con escasa probabilidad de éxito
12. y 13. Algunos monemas tradicionalmente vinculados a los remates, han adquirido particular recurrencia placas suspendidas a modo de esoberadon. Obedeciendo a distintos fines funcionales u
ornamentales, de protección (aletones) $o$ de resolución constructiva (ftontones), sus nuevas versiones, en general, reformulan las proporciones modelicas y se articulan o destacan con fines real innovación, por cuanto han permanecido a nivel de transtormación de lenguajes extranjeros Alerones y otros modelos se adaptan no siempre con el éxito estético que logra este caso con comunicativo y decodificación social. Luego, para alcanzar «Identidad» como la hemos entendido -en el sentido de la vocación a producir objetos que xidentifiquen" a la mayoría-, quedaría a los arquitectos uno de dos caminos:

- o consideramos la posibilidad de hacer una arquitectura que acoja la vocación vivencial y valórica del chileno común:

- o establecemos la comunicación mediante convergencias negativas (el j«feísmon!), lo cual está dentro de lo poco que concita un nivel de efectiva comunicación.

Más bien parece oportuno estimular la valoración social positiva de nuestras obras mediante el uso persuasivo del lenguaje arquitectónico, a partir de mensajes que contemplen códigos para una interpretación más cierta, buscando una nueva acepción al concepto de "viabilidad»: la comunicación viable, para una «factible» Identidad. Puede ser posible. no obstante que el gran Profesor Roberto Dávila -el "Maestro" para generaciones de arquitectos-, decía que nuestros antepasados mapuches creaban «imbunches», especie de monstruos vivos mediante la mutilación o deformación artificial de niños, como conjuro al mal para atraer lo bueno y lo bello. Significa, decía, que "los chilenos tenemos en nuestra alma el 'imbunchismo', que los arquitectos debemos empeñarnos en vencer'. ¿Tal vez no es posible?
REFERENCIAS

1. Leteler, S.; ARRIBAS, M.I.; RUGiero, A.. Descripción semiótica del Perfil de Identidad Arquifectónica en Chile Central...; Proyecto 2. LETELIER, S.; ARRIBAS, M.I.; Rugiero, A arquitectónica para la zona de Chile centrals D.1. № 3 ; Proy. cit.; enero 1995 3. AGUIRRE SILVA, J. «Hitos en Santiago. Esplendor decadencia en su Arquitectura y Paisajem, Publ.. C. Cordillera / DIEHGO; Viña del Mar, 1985 4. VAISMAN, L.,Semiologia Arquitectónica, D.D. AU., Universidad de Chile, Stgo. 1974

Nota:

Este artículo corresponde a las conclusiones de primer estudio sistemático sobre Identidad Arquitectonica que interpreta los hallazgos emanados en 1995 de la investigación FONDECYT 0805, sin antecedente de otras similares, realizado con las arquitectas y académicas $M$. Inés Arribas y Ana Rugiero como co-investigadoras. 